\title{
TU/e EmonONEN

\section{A negative bendixson-like criterion for a class of hybrid systems}

\section{Citation for published version (APA):}

Pogromski, A. Y., Nijmeijer, H., \& Rooda, J. E. (2007). A negative bendixson-like criterion for a class of hybrid systems. IEEE Transactions on Automatic Control, 52(4), 586-595. https://doi.org/10.1109/TAC.2007.894508

DOI:

10.1109/TAC.2007.894508

Document status and date:

Published: 01/01/2007

\section{Document Version:}

Publisher's PDF, also known as Version of Record (includes final page, issue and volume numbers)

\section{Please check the document version of this publication:}

- A submitted manuscript is the version of the article upon submission and before peer-review. There can be important differences between the submitted version and the official published version of record. People interested in the research are advised to contact the author for the final version of the publication, or visit the $\mathrm{DOI}$ to the publisher's website.

- The final author version and the galley proof are versions of the publication after peer review.

- The final published version features the final layout of the paper including the volume, issue and page numbers.

Link to publication

\section{General rights}

Copyright and moral rights for the publications made accessible in the public portal are retained by the authors and/or other copyright owners and it is a condition of accessing publications that users recognise and abide by the legal requirements associated with these rights.

- Users may download and print one copy of any publication from the public portal for the purpose of private study or research.

- You may not further distribute the material or use it for any profit-making activity or commercial gain

- You may freely distribute the URL identifying the publication in the public portal.

If the publication is distributed under the terms of Article 25fa of the Dutch Copyright Act, indicated by the "Taverne" license above, please follow below link for the End User Agreement:

www.tue.nl/taverne

Take down policy

If you believe that this document breaches copyright please contact us at:

openaccess@tue.nl

providing details and we will investigate your claim. 


\title{
A Negative Bendixson-Like Criterion for a Class of Hybrid Systems
}

\author{
Alexander Pogromsky, Henk Nijmeijer, Fellow, IEEE, and Jacobus Rooda, Member, IEEE
}

\begin{abstract}
A condition which ensures the absence of periodic orbits for nonsmooth dynamical systems is presented. The condition is a higher dimensional generalization of Bendixson's criterion applicable to differential inclusions that are useful in the description of hybrid systems. The main argument is based on contraction analysis of the $d$-measured volume along the system trajectories. A connection to methods for estimating the Hausdorff dimension is emphasized. For a class of hybrid systems described by a linear system with relay feedback the conditions are presented in the form of linear matrix inequalities. A simple but illustrative example is analyzed.
\end{abstract}

Index Terms-Differential inclusions, periodic solutions.

\section{INTRODUCTION}

D ISCONTINUOUS dynamical systems and, particularly, relay systems have attracted considerable attention over the last decades. While the mathematics of smooth dynamical systems still produces new and interesting discoveries, in applied disciplines it has been realized that for many applications discontinuities should be taken into account. For example, discontinuities can be used to simplify modeling of friction in mechanical systems, to design disturbance tolerant sliding mode controllers, to deal with a switching control strategy in manufacturing systems, and so on. A hot topic in research in the control community is formed by the class of so called hybrid dynamical systems, which combine continuous and discrete dynamics. Although the existing literature on this subject includes a growing number of monographs and papers (see, e.g., [1]-[5], to mention a few), those systems are far from being well understood.

Hybrid systems, being nonlinear dynamical systems, can have a very rich behavior and one of the main theoretical problems is to predict and to understand them without explicitly solving the equations describing the system. In this paper we study oscillatory behavior in nonlinear hybrid dynamical systems that recently receive lots of attention in the control community [6], [7]. The class of systems we study is

\footnotetext{
Manuscript received June 7, 2004; revised February 27, 2006 an July 10, 2006. Recommended by Associate Editor A. Bemporad. This work was supported in part by the EU projects SICONOS (IST-2001-37172), the HyCon Network of Excellence (contact number FP6-IST-511368), and the Dutch-Russian program on interdisciplinary mathematics "Dynamics and Control of Hybrid Mechanical Systems" (NWO Grant 047.017.018). A preliminary version of the paper was presented at the MTNS'04 mini-symposium "Mathematical theory of oscillations in networks and systems," Leuven, Belgium, 2004.

The authors are with the Department of Mechanical Engineering, Eindhoven University of Technology, Eindhoven 5600MB, The Netherlands (e-mail: a.pogromsky@tue.nl; h.nijmeijer@tue.nl; j.e.rooda@tue.nl).

Color versions of one or more of the figures in this paper are available online at http://ieeexplore.ieee.org.

Digital Object Identifier 10.1109/TAC.2007.894508
}

described by nonlinear differential equations with a discontinuous right-hand side. A particular result that is obtained here is a generalization of Bendixson's negative divergency test for that class of systems. This simple test gives a sufficient condition for the nonexistence of periodic orbits for smooth planar systems. This classical result claims that if in a simply connected domain the divergency of a vector field does not change sign, then this domain does not contain a periodic orbit. A classical proof of this statement is based on the divergence theorem and cannot be generalized to the higher dimensional case. The main purpose of this paper is to present a possible generalization of the Bendixson result to arbitrary dimension taking into account the possible discontinuity of the right hand side. There are several higher dimensional generalizations of this criterion, see, e.g., [8]-[12]. Muldowney and Li [9]-[11] used an approach based on compound matrices to prove a negative Bendixson-like criterion. In this paper we investigate this question by a method which allows to estimate the Hausdorff dimension of invariant compact sets [13]-[17]. In doing so, we first present a generalization for an estimate of the Hausdorff dimension formula for nonsmooth systems and then, based on that result we prove a negative criterion for the nonexistence of periodic orbits. It is also worth mentioning that although we present only sufficient conditions for nonexistence of periodic solutions of particular hybrid systems our approach is also applicable to disprove some bifurcations that can occur in such systems.

From a practical point of view a design based on global stability of a system can be too restrictive and conservative. A possible weaker criterion is that all trajectories tend to a set consisting of equilibrium points; that is the system can not exhibit oscillatory behavior. This fact indicates the importance of Bendixson-like criteria for the design and control of dynamical systems. A similar motivation can be found, for example in a recent paper [18], where for smooth systems a condition was presented which guarantees that almost all trajectories tend to an equilibrium. Another approach to simplify the stability analysis of discontinuous systems is based on the generalization of the Invariance Principle for differential inclusions, see, e.g., [19].

A general idea behind the proof of the nonexistence criterion is relatively simple. If one is able to show that in a simply connected positively invariant domain the (semi)flow generated by the system contracts the area of some initial surface, it is sufficient to claim that no periodic orbits can lie inside this domain. By reversing time, the same holds true for area-expanding systems. Together with Liouville's theorem this argument gives another proof of Bendixson's criterion that can be generalized to arbitrary dimensions. To characterize the area of a surface one can use the so called Hausdorff 2-measure, so area-contracting 


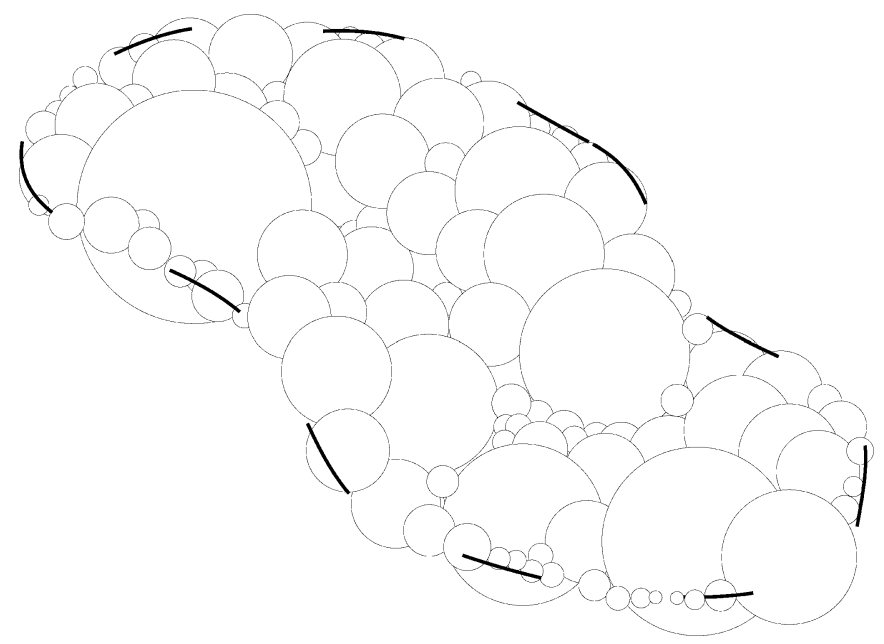

Fig. 1. Hausdorff 2-measure is approximated by the cross-sections of little balls with the surface.

systems are those for which the Hausdorff 2-measure of any initial measurable set vanishes with time.

The main method employed in our study is based on stability/ dichotomy-like properties of solutions with respect to each other rather than with respect to some invariant sets. The first results in this direction were developed by Demidovich [20], see also [21], [22] and Yoshizava [23]. Methods based on similar ideas are appreciated now in the control community [24]-[26]. A natural way to investigate those properties is based upon linearization of the dynamical system along any given trajectory which excludes the consideration of non smooth systems. In this paper, instead of linearization we investigate the behavior of some quadratic forms defined for a pair of trajectories of the system, and which allows to consider discontinuous systems. The conditions presented in this paper are formulated in terms of inequalities involving two eigenvalues of some matrix pencil.

The paper is organized as follows. In Section II, we present some necessary background material. Section III contains some result on the estimation of the Hausdorff dimension of invariant sets. Based on these results in Section IV we present a new version of a generalized Bendixson's criterion. Particular attention is then drawn to LMI-based results for linear systems with relay feedback.

\section{HAUSDORFF DIMENSION}

Consider a compact subset $K$ of $\mathbb{R}^{n}$. Given $d \geq 0, \varepsilon>0$, consider a covering of $K$ by open spheres $B_{i}$ with radii $r_{i} \leq \varepsilon$ (see Fig. 1). Denote by

$$
\mu(K, d, \varepsilon)=\inf \sum_{i} r_{i}^{d}
$$

the $d$-measured volume of covering of the set $K$. Here, the infimum is calculated over all $\varepsilon$-coverings of $K$. There exists a limit, which may be infinite

$$
\mu_{d}(K):=\sup _{\varepsilon>0} \mu(K, d, \varepsilon)=\lim _{\varepsilon \rightarrow 0} \mu(K, d, \varepsilon) .
$$

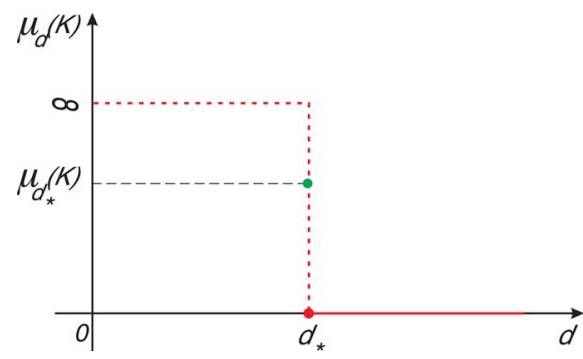

Fig. 2. Properties of the Hausdorff $d$-measure.

It can be proved that $\mu_{d}$ is a Borel regular measure on $\mathbb{R}^{n}$ (see [34]).

Definition 1: The measure $\mu_{d}$ is called the Hausdorff $d$-measure.

Some properties of the measure $\mu_{d}$ can be summarized as follows. There exists a single value $d=d_{*}$, such that for all $d<d_{*}, \mu_{d}(K)=+\infty$ and for all $d>d_{*}, \mu_{d}(K)=0$, with

$$
d_{*}=\inf \left\{d: \mu_{d}(K)=0\right\}=\sup \left\{d: \mu_{d}(K)=+\infty\right\} .
$$

(see [16, Prop. 5.3.2]).

Definition 2: The value $d_{*}$ is called the Hausdorff dimension of the set $K$.

In the sequel, we will use the notation $\operatorname{dim}_{H} K$ for the Hausdorff dimension of the set $K$.

For the control community the notions of Hausdorff measure and Hausdorff dimension are not common and we like to clarify the above definitions.

Suppose we have a two-dimensional bounded surface $S$ with area $m(S)$. We cover this surface by open spheres as required in the definition of the Hausdorff measure. Then, for $d=1$ and $d=3$ we have

$$
\begin{aligned}
& \mu_{1}(S)=\lim _{\varepsilon \rightarrow 0} \mu(S, 1, \varepsilon)=+\infty \\
& \mu_{3}(S)=\lim _{\varepsilon \rightarrow 0} \mu(S, 3, \varepsilon)=0
\end{aligned}
$$

while for $d=2$ we have

$$
\mu_{2}(S)=\frac{m(S)}{\pi}
$$

This example illustrates the behavior of $\mu_{d}(K)$ for a given $K$ as a function of $d$. Namely, for values of $d$ less than $\operatorname{dim}_{H} K, \mu_{d}(K)$ is infinite and for all values of $d$ greater than $\operatorname{dim}_{H} K \mu_{d}(K)$ is zero (see [16, Prop. 5.3.2]). This situation is schematically presented in Fig. 2.

For "good" sets such as a piece of an arc, a piece of a smooth surface, etc. the Hausdorff dimension can be used as the dimension in a normal sense (i.e., in the sense of Brouwer, or Lebesgue). This follows from the result (see, e.g., [16, Prop. 5.3.5]) which claims that for a set $K$ of positive $n$-dimensional Lebesgue measure the Hausdorff dimension of $K$ is $n$. However for other sets such as the Cantor set the value of the Hausdorff dimension can be fractional. Sets of such type are often encountered as invariant sets of "chaotic" systems, that makes the Hausdorff dimension of invariant sets an important characteristic for "chaotic" systems. 


\section{UPPER ESTIMATES FOR THE HAUSDORFF DIMENSION OF INVARIANT COMPACT SETS}

The extremal property of the Hausdorff dimension suggests an idea of how to estimate it for invariant sets of dynamical systems. Namely, if one is able to prove that for a given set $K$ its Hausdorff $d$-measure is zero, then it follows that $d$ is an upper estimate of the Hausdorff dimension of $K$. A possible way to show that the Hausdorff $d$-measure is zero is to prove the following inequality:

$$
\mu_{d}(\varphi(K)) \leq \nu \mu_{d}(K)
$$

where $\nu<1, \varphi$ is some mapping and $K$ an invariant set under $\varphi$, i.e., $\varphi(K)=K$, and hence $\mu_{d}(\varphi(K))=\mu_{d}(K)$. This identity together with (2) implies that $\mu_{d}(K)=0$.

When $\varphi$ is a flow generated by a system of differential equations inequality (2) follows from the fact that the $d$-measured volume of an open neighborhood of the invariant set $K$ decreases along the system trajectories. This observation suggests to employ a Lyapunov-like technique to estimate the Hausdorff dimension of invariant sets.

As has been mentioned in the Introduction, a generalization of Bendixson's criterion can be derived if one is able to show that in some simply connected region there are no invariant sets with Hausdorff dimension larger than or equal 2. This result can be obtained if one takes a 2-measured volume as a Lyapunov-like function candidate. However, in this section we present a more general result which holds for an arbitrary $d$-measure and in the next section we present a higher dimensional generalization of Bendixson's criterion.

Consider a system of differential equations

$$
\dot{x}=f(x) x \in \Omega \subset \mathbb{R}^{n} x_{0} \in \Omega
$$

where $f: \Omega \rightarrow \mathbb{R}^{n}$ is a (possibly) discontinuous vector field defined on some open positively invariant set $\Omega$, and which satisfies conditions guaranteeing the existence of solutions $x\left(t, x_{0}\right)$ in $\Omega$ in some reasonable sense, that is, if the function $f$ is discontinuous and satisfies some mild regularity assumptions, one can construct a set-valued function $\mathbf{f}$ according to numerous possible definitions (e.g., Filippov convex definition, Utkin's equivalent control, etc.) such that an absolutely continuous solution of the differential inclusion

$$
\dot{x} \in \mathbf{f}(x)
$$

is called a solution for system (3). We assume that the setvalued function $\mathbf{f}$ is bounded, upper semicontinuous with closed convex values.

The parameterized mapping $x_{0} \mapsto x\left(t, x_{0}\right), t \geq 0$, or the semi-flow will be denoted as $\varphi^{t}: \Omega \rightarrow \Omega$.

Consider a scalar differentiable function $V: \Omega \times \Omega \rightarrow \mathbb{R}$, $V(x, x)=0$.

Define the time derivative of the function $V$ along two solutions $x\left(t, x_{1}\right), x\left(t, x_{2}\right)$ of (3) as follows:

$$
\dot{V}\left(x_{1}, x_{2}\right):=\frac{\partial V\left(x_{1}, x_{2}\right)}{\partial x_{1}} \dot{x}_{1}+\frac{\partial V\left(x_{1}, x_{2}\right)}{\partial x_{2}} \dot{x}_{2}
$$

Since $V$ is Lipschitz continuous and the solutions $x\left(t, x_{i}\right)$ are absolutely continuous functions of time, the derivative

$$
\dot{V}\left(x\left(t, x_{1}\right), x\left(t, x_{2}\right)\right)
$$

exists almost everywhere in $\left[0, \min _{i} \bar{T}_{i}\right)$, where $\bar{T}_{i}$ is the maximal interval of existence of the solution $x_{i}\left(t, x_{i 0}\right)$ in $\Omega$.

For the function $V$, we can also define its upper derivative as follows:

$$
\dot{V}^{*}\left(x_{1}, x_{2}\right)=\sup _{\xi_{i} \in \mathbf{f}\left(x_{i}\right)}\left(\frac{\partial V\left(x_{1}, x_{2}\right)}{\partial x_{1}} \xi_{1}+\frac{\partial V\left(x_{1}, x_{2}\right)}{\partial x_{2}} \xi_{2}\right) .
$$

Then, for almost all $t \geq 0$ it follows that

$$
\dot{V}\left(x\left(t, x_{1}\right), x\left(t, x_{2}\right)\right) \leq \dot{V}^{*}\left(x\left(t, x_{1}\right), x\left(t, x_{2}\right)\right) .
$$

By the same token, the lower derivative of the function $V$ is defined as

$$
\dot{V}_{*}\left(x_{1}, x_{2}\right)=\inf _{\xi_{i} \in \mathbf{f}\left(x_{i}\right)}\left(\frac{\partial V\left(x_{1}, x_{2}\right)}{\partial x_{1}} \xi_{1}+\frac{\partial V\left(x_{1}, x_{2}\right)}{\partial x_{2}} \xi_{2}\right) .
$$

and satisfies

$$
\dot{V}\left(x\left(t, x_{1}\right), x\left(t, x_{2}\right)\right) \geq \dot{V}_{*}\left(x_{1}\left(t, x_{1}\right), x_{2}\left(t, x_{2}\right)\right) \text {. }
$$

almost everywhere.

We formulate the following hypothesis.

H1: There exists a $n \times n$ symmetric positive-definite matrix $P$, such that the function

$$
V\left(x_{1}, x_{2}\right)=\left(x_{1}-x_{2}\right)^{\top} P\left(x_{1}-x_{2}\right)
$$

satisfies the following inequality:

$$
\dot{V}^{*}\left(x_{1}, x_{2}\right) \leq\left(x_{1}-x_{2}\right)^{\top} Q\left(x_{1}\right)\left(x_{1}-x_{2}\right)+o\left(\left\|x_{1}-x_{2}\right\|^{2}\right)
$$

for all $x_{1}, x_{2} \in \Omega$ with a symmetric differentiable matrix valued function $Q$, bounded on $\Omega$ and with the function of higher order terms $O$ obeying

$$
\frac{o\left(\left\|x_{1}-x_{2}\right\|^{2}\right)}{\left\|x_{1}-x_{2}\right\|^{2}} \rightarrow 0, \text { as }\left\|x_{1}-x_{2}\right\| \rightarrow 0
$$

uniformly over $x_{1}, x_{2}$ from any compact subset of $\Omega$.

Hla: The lower derivative of the function $W\left(x_{1}, x_{2}\right)=$ $\left(x_{1}-x_{2}\right)^{\top} Q\left(x_{1}\right)\left(x_{1}-x_{2}\right)$ satisfies the following condition: For any compact subset of $\Omega$ there is a number $M$ that

$$
\dot{W}_{*}\left(x_{1}, x_{2}\right) \geq M V\left(x_{1}, x_{2}\right) \text {. }
$$

Note that assumption H1a is always satisfied if $f$ is locally Lipschitz continuous.

$H 2$ : All solutions starting in $\Omega$ are defined for all $t \geq 0$.

We begin with the following preliminary result.

Lemma 1: Suppose Assumptions $\mathrm{H} 1$ and $\mathrm{H} 2$ are satisfied. Then any solution $x\left(t, x_{0}\right)$ to (3), with $x_{0} \in \Omega$ is right-unique 
(for the definition of right-uniqueness see [1, Ch. 2, p. 106]) and depends continuously on the initial conditions.

Proof: We prove the statement under the weaker assumption that for all $x_{1}, x_{2}$ from any compact subset of $\Omega$ there is a positive $L$ such that

$$
\dot{V}^{*} \leq L\left(x_{1}-x_{2}\right)^{\top} P\left(x_{1}-x_{2}\right) .
$$

Then

$$
\frac{d}{d t}\left(V\left(x_{1}(t), x_{2}(t)\right) e^{-L t}\right) \leq 0
$$

almost everywhere. The absolutely continuous function $V e^{-L t}$ does not increase, and if $x_{1}=x_{2}$ it follows that $V\left(x_{1}(t), x_{2}(t)\right)=0$ for $t \geq 0$ where $x_{i}(t)=x\left(t, x_{i}\right)$. Thus the right-uniqueness is proved. The local Lipschitz continuous dependence on the initial conditions follows from the inequalities:

$$
\begin{aligned}
\left\|x_{1}(t)-x_{2}(t)\right\|^{2} & \leq \frac{1}{\lambda_{\min }(P)} V\left(x_{1}(t), x_{2}(t)\right) \\
& \leq \frac{1}{\lambda_{\min }(P)} V\left(x_{1}, x_{2}\right) e^{L t} \\
& \leq \frac{\lambda_{\max }(P)}{\lambda_{\min }(P)} e^{L t}\left\|x_{1}-x_{2}\right\|^{2}
\end{aligned}
$$

where $\lambda_{\min }(P)$ and $\lambda_{\max }(P)$ are the lower and upper bounds for the eigenvalues of $P$.

The previous lemma shows that the Cauchy problem (3) is well-posed and continuous dependence on initial conditions follows.

Let $\lambda_{1}(x) \geq \lambda_{2}(x) \geq \cdots \geq \lambda_{n}(x), x \in \Omega$ be the ordered solutions of the following generalized eigenvalue problem:

$$
\operatorname{det}(Q(x)-\lambda P)=0
$$

which are real since both $Q$ and $P$ are symmetric.

Consider a compact set $S$ of finite Hausdorff $d$-measure for some $d=d_{0}+s, d \leq n$, where $d_{0} \in \mathbb{N}$ and $s \in[0,1)$. Suppose that $S \subset \Omega$, then $\varphi^{t}(S) \subset \Omega$ for all positive $t$. Now, we formulate the following result.

Theorem 1: Suppose hypotheses $\mathrm{H} 1, \mathrm{H} 1 \mathrm{a}$, and $\mathrm{H} 2$ are satisfied. If for some $d=d_{0}+s, 0<d_{0} \leq n, 0 \leq s<1$ it follows that

$$
\sup _{x \in \Omega}\left(\lambda_{1}(x)+\cdots+\lambda_{d_{0}}(x)+s \lambda_{d_{0}+1}(x)\right)<0 .
$$

Then

$$
\lim _{t \rightarrow \infty} \mu_{d}\left(\varphi^{t}(S)\right)=0
$$

The proof is based on the construction of a finite set of affine maps $\mathbb{R}^{n} \rightarrow \mathbb{R}^{n}$ which locally approximate $\varphi^{t}$. Then, using the linear part of those maps we approximate how the $d$-measured volume is changed under those maps to compute the change of $\mu_{d}\left(\varphi^{t}(S)\right)$. The proof of this theorem is presented in the Appendix .
The main result of this section is the following theorem.

Theorem 2: Suppose hypotheses $\mathrm{H} 1, \mathrm{H} 1 \mathrm{a}$, and $\mathrm{H} 2$ are satisfied, and there exist positive integer $d_{0}$ and real $s \in[0,1)$ such that

$$
\sup _{x \in \Omega}\left(\lambda_{1}(x)+\cdots+\lambda_{d_{0}}(x)+s \lambda_{d_{0}+1}(x)\right)<0 .
$$

Suppose that there is an invariant compact set $K \in \Omega$.

Then, $\operatorname{dim}_{H} K \leq d_{0}+s$.

Proof: From the previous result it follows that $\mu_{d}\left(\varphi^{t}(K)\right) \rightarrow 0$ as $t \rightarrow \infty$. Since $K$ is invariant $\mu_{d}(K)=\mu_{d}\left(\varphi^{t}(K)\right)$. Therefore, $\mu_{d}(K)=0$.

For the continuously differentiable right-hand side of system (3) the previous theorem can be formulated in infinitesimal form.

Corollary 1: [17] Suppose that hypothesis $\mathrm{H} 2$ is satisfied and there is an invariant compact set $K \in \Omega$. Assume also that there is a symmetric positive definite $n \times n$ matrix $P$ such that the solutions of the following generalized eigenvalue problem:

$$
\operatorname{det}(Q(x)-\lambda P)=0
$$

satisfy the inequality (6), with the matrix $Q$ defined as

$$
Q=P \frac{\partial f(x)}{\partial x}+\frac{\partial f^{\top}(x)}{\partial x} P .
$$

Then, $\operatorname{dim}_{H} K \leq d_{0}+s$.

In [17], this result was derived using the linearization of the flow $\varphi^{t}$ with an approach close to that due to Douady-Oesterlé [13] and Leonov[16]. It is now seen that this result can also be obtained from a more general argument which is applicable to discontinuous systems.

We have presented local conditions that are easier to verify analytically for particular examples. One can derive a further generalization via integral conditions of the form

$$
\sup _{x_{10}} \int_{0}^{\tau}\left(\lambda_{1}\left(x\left(t, x_{10}\right)\right)+\cdots+s \lambda_{d_{0}+1}\left(x\left(t, x_{10}\right)\right)\right) d t<0
$$

which can be useful for numerical methods.

\section{A. Example: The Lorenz System}

It is well known that all trajectories of the Lorenz system

$$
\left\{\begin{array}{l}
\dot{x}=\sigma(y-x) \\
\dot{y}=r x-y-x z \\
\dot{z}=-b z+x y
\end{array}\right.
$$

are ultimately bounded for arbitrary positive $\sigma, r, b$; that is, for fixed $\sigma, r, b$ there is an invariant compact set $K$. Let us estimate its Hausdorff dimension.

Proposition 1: Suppose the parameters of the system are such that the following inequality:

$$
\limsup _{t \rightarrow \infty}\left[\frac{y(t)^{2}}{b}+(z(t)-2 r)^{2}\right] \leq 4 r^{2} .
$$




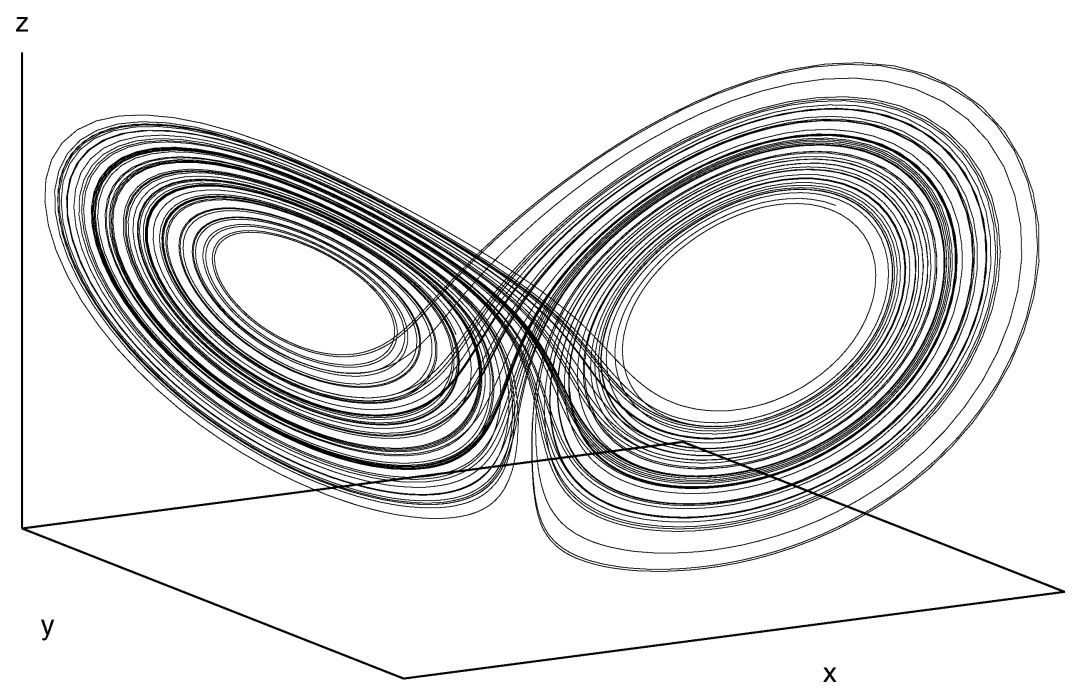

Fig. 3. Typical trajectory of the Lorenz system.

is satisfied for all initial conditions. Then

$$
\operatorname{dim}_{H} K \leq 3-\frac{2(\sigma+b+1)}{\sigma+1+\sqrt{(\sigma-1)^{2}+4 r \sigma}} .
$$

Proof: The Jacobian of the Lorenz system is

$$
J=\left(\begin{array}{ccc}
-\sigma & \sigma & 0 \\
r-z & -1 & -x \\
y & x & -b
\end{array}\right)
$$

and let $P$ be

$$
P=\left(\begin{array}{ccc}
\frac{r}{\sigma} & 0 & 0 \\
0 & 1 & 0 \\
0 & 0 & 1
\end{array}\right)
$$

then

$$
P J+J^{\top} P=\left(\begin{array}{ccc}
-2 r & 2 r-z & y \\
2 r-z & -2 & 0 \\
y & 0 & -2 b
\end{array}\right) .
$$

We know that $\lambda_{1}+\lambda_{2}+\lambda_{3}=-2(\sigma+1+b)$, and from $-2(\sigma+$ $1+b)<0$ it follows that $\lambda_{3}<0$ for all $x, y, z$. Hence, by Corollary 1 , to find an upper estimate of $s$ it is sufficient to find a lower estimate of $\bar{\lambda}_{3}$. Given this estimate, we then evaluate the upper bound on the Hausdorff dimension as

$$
\operatorname{dim}_{H} K \leq 3-\frac{2(\sigma+b+1)}{\left|\bar{\lambda}_{3}\right|}
$$

The smallest negative eigenvalue of some symmetric matrix $Q$ is a number $\bar{\lambda}$ such that the matrix $Q-\lambda I$ is positive definite as long as $\lambda<\bar{\lambda}$. For all nonpositive $\lambda$ the following matrix inequality:

$$
P J+J^{\top} P-\lambda P \geq P J+J^{\top} P-\lambda P_{1}
$$

is satisfied, where $P_{1}=\operatorname{diag}\{r / \sigma, 1,0\}$. Therefore, to find a lower estimate for $\lambda_{3}$ it is sufficient to find a lower estimate of the smallest nonpositive solution of the following equation:

$$
\operatorname{det}\left(P J+J^{\top} P-\lambda P_{1}\right)=0
$$

or

$$
r\left(2+\frac{\lambda}{\sigma}\right)(2+\lambda)=\frac{y^{2}}{b}+(2 r-z)^{2}+\lambda \frac{y^{2}}{2 b} .
$$

Using inequality (8) and neglecting the term $\lambda y^{2} / 2 b$ (since we are looking for a negative lower estimate of the smallest solution for $\lambda$ ) it is then straightforward to complete the proof.

In [27], it has been proven that for the standard values of parameters of the Lorenz system $\sigma=10, r=28, b=8 / 3$, the inequality (8) is satisfied. Thus, in this case, $\operatorname{dim}_{H} K \leq 2.4013$. A typical trajectory of the Lorenz system for those values of the parameters is presented in Fig. 3.

The inequality (9) claims that the Hausdorff dimension of the invariant set of the (chaotic) Lorenz system is bounded by the Lyapunov dimension of the origin. This statement is known as the Eden conjecture [28] which has been proved by Ljashko [29] for a certain set of parameters. The method proposed by Ljashko does not depend on the estimates of the ultimate bounds for the system trajectories but leads to very cumbersome calculations. Recently, Leonov found a set of parameters of the Lorenz system for which the Lyapunov dimension of the Lorenz attractor equals to the local Lyapunov dimension of the origin [30]. Our result can be proved with a much simpler derivation.

\section{A Higher Dimensional Generalization OF BENDIXSON'S CRITERION}

We begin with some definitions.

Definition 3: [33] A set $S \subset \mathbb{R}^{n}$ is called $d$-dimensional rectifiable set, $d \in \mathbb{N}$ if $\mu_{d}(S)<\infty$ and $\mu_{d}$-almost all of $S$ is contained in the union of the images of countably many Lipschitz functions from $\mathbb{R}^{d}$ to $\mathbb{R}^{n}$.

The rectifiable sets are generalized surfaces of geometric measure theory. Any one-dimensional closed rectifiable contour 
$\gamma$ bounds some two-dimensional rectifiable set, for example the cone over $\gamma$.

A set is said to be simply connected if any simple closed curve can be contracted to a point continuously in the set.

Theorem 3: Suppose that Assumptions H1, H1a, and $\mathrm{H} 2$ are satisfied, let $\Omega$ be a simply connected set. Suppose that

$$
\sup _{x \in \Omega} \lambda_{1}(x)+\lambda_{2}(x)<0 .
$$

Then, $\Omega$ does not contain whole periodic orbits.

Proof: The proof of Theorem 3 follows an idea used in the proof of the Leonov theorem [32], see also [31, Th. 8.3.1]. Suppose (10) holds but there is a periodic orbit $\gamma$ passing through a point $x_{0} \gamma:=\left\{x \mid \exists t \geq 0, x=x\left(t, x_{0}\right)\right\}$ which lies entirely in $\Omega$.

Since as assumed the function $\mathbf{f}$ is bounded on any compact set it follows that there is a positive constant $L>0$ such that for all $x_{0} \in \gamma$

$$
\left\|x\left(t_{1}, x_{0}\right)-x\left(t_{2}, x_{0}\right)\right\| \leq L\left|t_{1}-t_{2}\right|
$$

and thus the set $\gamma$ is the image of a Lipschitz continuous function. Therefore, the set $\gamma$ is a rectifiable one-dimensional set. From the theorem on existence of area-minimizing surfaces (see $[34$, Th. 5.6]) it follows that there exists a two-dimensional rectifiable set $\bar{S} \in \mathbb{R}^{n}$ such that its boundary is $\gamma$ and it has minimal Hausdorff 2-measure.

Let $S$ be a rectifiable two-dimensional set $S \subset \Omega$ with boundary $\gamma$. The existence of such set follows from the fact that $\Omega$ is simply connected. As before, we denote by $\varphi^{t}$ the flow of system (3). Let $\mu(S)$ be the Hausdorff 2-measure of a 2-dimensional surface $S$. Since $\gamma$ is invariant under $\varphi^{t}$ and $\varphi^{t}(S) \subset \Omega$ for any $t \geq 0$ ( $\Omega$ is positively invariant) we have

$$
\inf _{t \geq 0} \mu\left(\varphi^{t}(S)\right) \geq \mu(\bar{S})>0
$$

At the same time, using (10) from Theorem 1 it follows that

$$
\lim _{t \rightarrow \infty} \mu\left(\varphi^{t}(S)\right)=0
$$

which contradicts (11). Therefore, (10) ensures the absence of periodic trajectories lying in $\Omega$.

It is worth noting that this theorem being applied to smooth systems together with its time reversed version (for smooth systems we have local right and left uniqueness) gives the classical Bendixson divergency condition.

The main idea of the proof (see [32]) is based on the existence of a surface with minimal area given its boundary. Although the mathematical problem of proving existence of a surface that has minimal area and is bounded by a prescribed curve, has a long defied mathematical analysis, an experimental solution is easily obtained by a simple physical device. Plateau, a Belgian physicist, studied the problem by dipping an arbitrarily shaped wire frame into a soap solution. The resulting soap film corresponds to a relative minimum of area and thus produces a minimal surface spanned by that wire contour. A classical solution to Plateau's problem can be found, for example, in [35] with some regularity assumptions on the contour $\gamma$ that can be violated if $\gamma$ is a closed orbit corresponding to a periodic solution of a system of differential equations with discontinuous right hand sides. Fortunately, the argument based on geometric measure theory allows to overcome this difficulty.

A final remark in this section is that the theorem can disprove not only the existence of periodic orbits, but also one-dimensional rectifiable invariant sets (see the proof). Therefore the main results of this section can be also used to disprove the existence of invariant sets of more general nature like homoclinic and/or heteroclinic orbits.

\section{A. Example}

Consider the following system:

$$
\dot{x}=A x+B u \quad u=-\operatorname{sign}(y) y=C x
$$

where $x \in \mathbb{R}^{3}, u, y \in \mathbb{R}^{1}$ and the matrices $A, B, C$ are given as follows:

$$
A=\left(\begin{array}{rrr}
\alpha & 1 & 1 \\
-1 & \beta & -1 \\
-1 & 1 & -1
\end{array}\right) \quad B=\left(\begin{array}{l}
0 \\
0 \\
b
\end{array}\right) \quad C=\left(\begin{array}{lll}
0 & 0 & 1
\end{array}\right)
$$

with positive $b$. Consider the smooth function (4) in the form

$$
V=\left(x_{1}-x_{2}\right)^{\top}\left(x_{1}-x_{2}\right) .
$$

For this system the corresponding solution according to the Filippov convex definition coincides with the Utkin solution [1]. At the discontinuity points of the right-hand side, the corresponding set valued function in the differential inclusion is obtained by the closure of the graph of the right hand side and by passing over to the convex hull. As shown in [1, p. 155], these procedures do not increase the upper value of $\dot{V}^{*}$ and hence it is sufficient to compute the derivative of $V$ only in the area of continuity of the right-hand side. The derivative of $V$ in this area satisfies

$$
\dot{V} \leq 2\left(x_{1}-x_{2}\right)^{\top}\left(\begin{array}{rrr}
\alpha & 0 & 0 \\
0 & \beta & 0 \\
0 & 0 & -1
\end{array}\right)\left(x_{1}-x_{2}\right) .
$$

The condition Hla is satisfied since $b>0$. The previous theorem suggests that if $\min \{\alpha, \beta\} \geq-1$, a sufficient condition for the absence of periodic solutions is

$$
\alpha+\beta<0 .
$$

To demonstrate that violation of the condition (14) can result in oscillatory behavior we perform a computer simulation for the following parameter values: $\alpha=1, \beta=-1 / 2, b=1$. The 


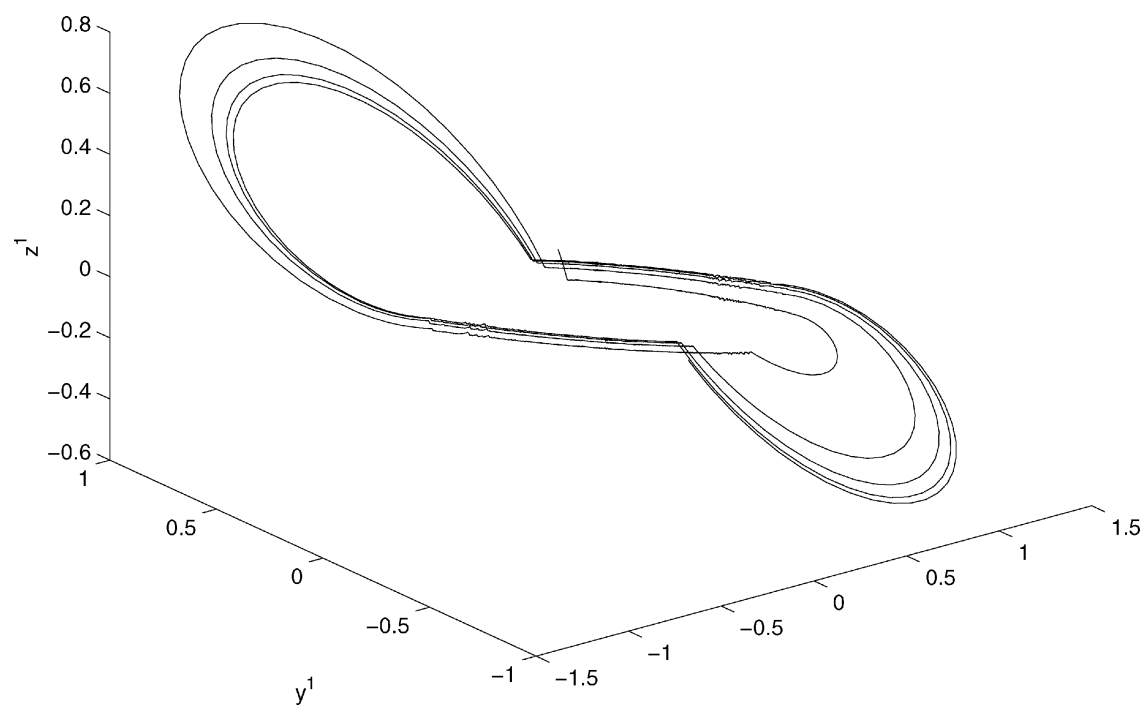

Fig. 4. Oscillatory behavior of (13) for $\alpha+\beta>0$.

results of the simulation are presented in Fig. 4. It is seen that the system possesses an orbitally stable limit cycle.

\section{B. An LMI Based Criterion for Lur'e Systems With Discontinuous Right-Hand Side}

In the previous example, the matrix $A$ was chosen as a sum of a diagonal and a skew-symmetric matrix that made all necessary calculations trivial. Next we present an LMI based criterion which ensures the absence of periodic solutions for the following system:

$$
\dot{x}=A x+B u \quad u=-b \operatorname{sign}(y) \quad y=C x
$$

where $x \in \mathbb{R}^{n}, n \geq 2, u, y \in \mathbb{R}^{1}, b>0$ and the matrices $A, B, C$ are of corresponding dimensions.

Theorem 4: Suppose there exists $\mu$ and positive definite matrix $P$ such that the following inequality:

$$
\left(\begin{array}{ccc}
P\left(A-\mu I_{n}\right)+\left(A-\mu I_{n}\right)^{\top} P & * & * \\
B^{\top} P-C & 0 & 0 \\
B^{\top}\left(P A+A^{\top} P\right)+\gamma C & 0 & 0
\end{array}\right) \geq 0
$$

is satisfied for some $\gamma \geq 0$. Then if

$$
\operatorname{tr} A-(n-2) \mu<0
$$

the system (15) does not have periodic solutions.

Proof: According to (16) the matrix $P$ satisfies the following equation $P B=C^{\top}$. Thus, taking the derivative of the following function:

$$
V=\left(x_{1}-x_{2}\right)^{\top} P\left(x_{1}-x_{2}\right)
$$

yields (as in the previous example it is sufficient to compute the derivative in the area of continuity of the right-hand side)

$$
\begin{aligned}
\dot{V}= & \left(x_{1}-x_{2}\right)^{\top}\left(P A+A^{\top} P\right)\left(x_{1}-x_{2}\right) \\
& -2 b\left(C x_{1}-C x_{2}\right)\left(\operatorname{sign} C x_{1}-\operatorname{sign} C x_{2}\right) \\
\leq & \left(x_{1}-x_{2}\right)^{\top}\left(P A+A^{\top} P\right)\left(x_{1}-x_{2}\right) .
\end{aligned}
$$

Let us verify the condition H1a with the function

$$
W=\left(x_{1}-x_{2}\right)^{\top}\left(P A+A^{\top} P\right)\left(x_{1}-x_{2}\right) .
$$

With similar calculations as above the function $W$ satisfies the condition H1a since $\left(P A+A^{\top} P\right) B=-\gamma C^{\top}$.

Now consider the smallest solution $\lambda_{n}$ of the following equation:

$$
\operatorname{det}\left(P A+A^{\top} P-\lambda P\right)=0
$$

From the hypothesis it follows that $\lambda_{n} \geq 2 \mu$. On the other hand if $\lambda_{i}, i=1, \ldots, n$ are the solutions of (18) then

$$
\lambda_{1}+\cdots+\lambda_{n}=2 \operatorname{tr} A \text {. }
$$

Since

$$
\lambda_{i} \geq \lambda_{n}
$$

it follows that

$$
\lambda_{1}+\lambda_{2} \leq 2(\operatorname{tr} A-(n-2) \mu)<0
$$

and according to Theorem 3 the system (15) has no periodic solutions.

\section{CONCLUSION}

In this paper we presented a new discontinuous version of a Bendixson's like criterion. The criterion is based on a new result on the estimation of the Hausdorff dimension of invariant sets for (possibly) discontinuous systems. The new criterion can be applied for the design and control of discontinuous systems when the requirement of global stability is too restrictive. Our study is based on dichotomy-like properties of solutions of dynamical systems with respect to each other rather than with respect to some invariant sets. We hope that further development 
of this approach will allow better understanding bifurcations in nonsmooth dynamical systems [37].

\section{APPENDIX}

\section{PROOF OF THEOREM 1}

We prove the theorem under condition that $P=I_{n}$ since this condition can be met under appropriate linear change of coordinates.

For a linear operator $T$ in $\mathbb{R}^{n}$ let $\sigma_{1} \geq \cdots \geq \sigma_{n}$ be its singular values and introduce the following notation for some $d=d_{0}+s$, $d \leq n, s \in[0,1) \omega_{d}(T)=\sigma_{1} \sigma_{d_{0}} \sigma_{d_{0}+1}^{s}$. If $d=0$ we put $\omega_{d}(T)=\sigma_{1}$.

For similarity denote

$$
x\left(t, x_{1}\right)=x_{1}(t), x\left(t, x_{2}\right)=x_{2}(t), \Delta(t)=x_{1}(t)-x_{2}(t) .
$$

Since $V\left(x_{1}(t), x_{2}(t)\right)$ is an absolutely continuous function of time

$$
\begin{aligned}
V\left(x_{1}(t), x_{2}(t)\right)-V\left(x_{1}, x_{2}\right)= & \int_{0}^{t} \dot{V}\left(x_{1}(\tau), x_{2}(\tau)\right) d \tau \\
\leq & \int_{0}^{t} \dot{V}^{*}\left(x_{1}(\tau), x_{2}(\tau)\right) d \tau \\
\leq & \int_{0}^{t} \Delta^{\top}(\tau) Q\left(x_{1}(\tau)\right) \Delta(\tau) d \tau \\
& +\int_{0}^{t} o\left(\left\|x_{1}(\tau)-x_{2}(\tau)\right\|^{2}\right) d \tau
\end{aligned}
$$

and, therefore

$$
\Delta^{\top}(t)\left(I_{n}-t Q\left(x_{1}(t)\right)\right) \Delta(t) \leq \Delta(0)^{\top} \Delta(0)+h
$$

where the higher order function $h$ is given by $h=h_{1}+h_{2}$ with

$$
h_{1}=\int_{0}^{t} \Delta^{\top}(\tau) Q\left(x_{1}(\tau)\right) \Delta(\tau) d \tau-t \Delta^{\top}(t) Q\left(x_{1}(t)\right) \Delta(t)
$$

and

$$
h_{2}=\int_{0}^{t} o\left(\left\|x_{1}(\tau)-x_{2}(\tau)\right\|^{2}\right) d \tau .
$$

Let us estimate $h_{1}$. According to the mean value theorem

$$
h_{1}=t\left[\Delta^{\top}(\theta t) Q\left(x_{1}(\theta t)\right) \Delta(\theta t)-\Delta^{\top}(t) Q\left(x_{1}(t)\right) \Delta(t)\right]
$$

for some $0<\theta<1$. Let $\mathcal{B}$ be a compact subset of $\Omega$ such that $\forall \tau, 0 \leq \tau \leq t, x_{1}(\tau), x_{2}(\tau) \in \mathcal{B}$. Denoting

$$
q:=t\left[\Delta^{\top}(\theta t) Q\left(x_{1}(\theta t)\right) \Delta(\theta t)-\Delta^{\top}(t) Q\left(x_{1}(t)\right) \Delta(t)\right]
$$

it follows from assumption H1a that

$$
\begin{aligned}
q & \leq-t M(\mathcal{B}) \int_{\theta t}^{t} \Delta^{\top}(\tau) \Delta(\tau) d \tau \\
& =-t^{2}(1-\theta) M(\mathcal{B}) \Delta^{\top}(\xi) \Delta(\xi)
\end{aligned}
$$

with $\theta t<\xi<t$. With the same $L$ as in the proof of Lemma 1

$$
\Delta^{\top}(\xi) \Delta(\xi) \leq e^{L(\mathcal{B}) \xi} \Delta^{\top}(0) \Delta(0)
$$

and, therefore

$$
\left|h_{1}\right| \leq t^{2}(1-\theta)|M(\mathcal{B})| e^{L(\mathcal{B}) \xi} \Delta^{\top}(0) \Delta(0) .
$$

Hence, if $x_{2}$ belong to the ball $E_{x_{1}}$ of radius $\varepsilon$ centered at $x_{1}$

$$
E_{x_{1}}=\left\{x \in \mathbb{R}^{n} \mid\left(x-x_{1}\right)^{\top}\left(x-x_{1}\right) \leq \varepsilon^{2}\right\}
$$

then $\left|h_{1}\right|=o(t) O\left(\varepsilon^{2}\right)$. Using the same ideas to estimate $h_{2}$ yields

$$
\left|h_{2}\right|=O(t) o\left(\varepsilon^{2}\right)
$$

and, finally

$$
|h|=o(t) O\left(\varepsilon^{2}\right)+O(t) o\left(\varepsilon^{2}\right) .
$$

According to (20) the mapping $\varphi^{t}$ maps the ball $E_{x_{1}}$ into the ellipsoid $E_{x_{1}}^{\prime}$

$$
\begin{array}{r}
E_{x_{1}(t)}^{\prime}=\left\{x \in \mathbb{R}^{n} \mid\left(x-x_{1}(t)\right)^{\top}\left(I-t Q\left(x_{1}(t)\right)\right)\left(x-x_{1}(t)\right)\right. \\
\left.\leq \varepsilon^{2}+|h|\right\} .
\end{array}
$$

To estimate the singular values of the linear part $T_{x}$ of $\varphi^{t}$, given $t>0$, consider any linear operator that maps $E_{x_{1}}$ into $E_{x_{1}}^{\prime \prime}$ that can be rewritten as

$$
\begin{aligned}
E_{x_{1}}^{\prime \prime} & =\left\{x \in \mathbb{R}^{n} \mid\left(x-x_{1}\right)^{\top} \mu\left(I-t Q\left(x_{1}(t)\right)\right)\left(x-x_{1}\right) \leq \varepsilon^{2}\right\} \\
\mu & =\frac{\varepsilon^{2}}{\varepsilon^{2}+|h|}=\frac{1}{1+\frac{|h|}{\varepsilon^{2}}} .
\end{aligned}
$$

This linear operator $T$, should satisfy the following relation for $\eta \in \mathbb{R}^{n}$

$$
\mu \eta^{\top} T^{\top}(I-t Q) T \eta \leq \eta^{\top} \eta
$$

Since for any square matrix $X$ the matrices $X^{\top} X$ and $X X^{\top}$ are orthogonally congruent, the last inequality can be rewritten as

$$
\mu S T T^{\top} S^{\top} \leq I
$$

where $S^{\top} S=I-t Q$ is a positive definite matrix provided $0<t<1 /\left|\lambda_{1}\right|$, with $\lambda_{1} \geq \cdots \geq \lambda_{n}$ being the eigenvalues of $Q$. The last inequality is equivalent to

$$
T T^{\top} \leq \frac{1}{\mu} S^{-1} S^{-\top}=\frac{1}{\mu}(I-t Q)^{-1}
$$


and using simultaneous diagonalization of the right- and lefthand sides of this inequality one gets the following estimate:

$$
\begin{aligned}
\omega_{d}(T) \leq & \left(1+\frac{|h|}{\varepsilon^{2}}\right)^{d} \\
& \times \frac{1}{\sqrt{\left(1-t \lambda_{1}\right) \cdots\left(1-t \lambda_{d_{0}}\right) \cdot\left(1-t \lambda_{d_{0}+1}\right)^{s}}} \\
= & \left(1+d \frac{|h|}{\varepsilon^{2}}+o\left(\frac{|h|}{\varepsilon^{2}}\right)\right) \\
& \times\left(1+\frac{t}{2}\left(\lambda_{1}+\cdots+\lambda_{d_{0}}+s \lambda_{d_{0}+1}\right)+o(t)\right) .
\end{aligned}
$$

Passing to the limit for $\varepsilon \rightarrow 0$ and using the condition imposed on $\lambda$ 's one concludes that there is a $t \geq 0$ such that $\omega_{d}\left(T_{x}\right) \leq \varrho<1$. Now, the proof can be completed along the same lines as in the proof of Douady and Osterlé result (see, e.g., [16, Th. 5.4.1]): As before, via the chain rule, the estimation of the singular values of the linear part $T_{x}^{m}$ of $\varphi^{m t}, m=1,2 \ldots$, satisfies

$$
\begin{aligned}
& \omega_{d}\left(T_{x}^{m}\right) \\
& =\omega_{d}\left(T_{\varphi_{x}^{(m-1) t}} \ldots T_{\varphi^{t} x} T_{x}\right) \leq \omega_{d}\left(T_{\varphi_{x}^{(m-1) t}}\right) \cdots \omega_{d}\left(T_{x}\right) \leq \varrho^{m}
\end{aligned}
$$

and the Hausdorff $d$-measure of $S$ decays with time.

\section{ACKNOWLEDGMENT}

The authors thank Prof. G.A. Leonov from St. Petersburg State University for fruitful discussions during his visit to the Eindhoven University of Technology.

\section{REFERENCES}

[1] A. F. Filippov, Differential Equations with Discontinuous Right Hand Sides. Dordrecht, The Netherlands: Kluwer, 1988.

[2] V. I. Utkin, Sliding Modes in Control Optimization. Berlin, Germany: Springer-Verlag, 1992.

[3] Y.Z. Tsypkin, Relay Control Systems. Cambridge, U.K.: Cambridge Univ. Press, 1984

[4] F. H. Clarke, Y. S. Ledyaev, R. J. Stern, and P. R. Wolenski, Nonsmooth Analysis and Control Theory. Berlin, Germany: Springer-Verlag, 1998.

[5] A. S. Matveev and A. V. Savkin, Qualitative Theory of Hybrid Dynamical Systems. Berlin, Germany: Birkhäuser, 2000.

[6] J. M. Goncalves, A. Megretski, and M. Dahleh, "Global stability of relay feedback systems," IEEE Trans. Autom. Control, vol. 46, no. 4, pp. 550-562, Apr. 2001.

[7] _ "Global analysis of piecewise linear systems using impact maps and surface Lyapunov functions," IEEE Trans. Autom. Control, vol. 48, no. 12, pp. 2089-2106, Dec. 2003.

[8] R. A. Smith, "An index theorem and Bendixon's negative criterion for certain differential equations of higher dimension," Proc. Roy. Soc. Edinburgh, vol. 91A, pp. 63-77, 1981.

[9] J. Muldowney, "Compound matrices and ordinary differential equations," Rocky Mountain J. Math., vol. 20, no. 4, pp. 857-871, 1990.

[10] Li, Yi, and J. S. Muldowney, "On Bendixson's criterion," J. Diff. Equat., vol. 106, pp. 27-39, 1993.

[11] — "Phase asymptotic semiflows, Poincare's condition, and the existence of stable limit cycles," J. Diff. Equat., vol. 124, pp. 425-448, 1996.

[12] K. E. Starkov, "Localization of periodic orbits of polynomial of even degree by linear functions vector fields," Chaos: Solitons Fractals, vol. 25 , no. 3, pp. 621-627, 2005.
[13] A. Douady and J. Osterlé, "Dimension de Hausdorff des attracteurs," C.R. Acad. Sci. Paris. Sér. A., vol. 290, no. 24, pp. 1135-1138, 1980.

[14] R. A. Smith, "Some applications of Hausdorff dimension inequalities for ordinary differential equations," Proc. Royal Soc. Edinburgh, vol. 104A, no. 3-4, pp. 235-259, 1986

[15] R. Témam, Infinite-Dimensional Dynamical Systems in Mechanics and Physics. New York: Springer-Verlag, 1988.

[16] G. A. Leonov, D. V. Ponomarenko, and V. B. Smirnova, Frequency Methods for Nonlinear Analysis. Theory and Applications. Singapore: World Scientific, 1996.

[17] A. Y. Pogromsky and H. Nijmeijer, "On estimates of Hausdorff dimension of invariant compact sets," Nonlinearity, vol. 13, pp. 927-945, 2000.

[18] A. Rantzer, "A dual to Lyapunov's stability theorem," Sys. Control Lett., vol. 42, pp. 161-168, 2001.

[19] J. Alvarez, I. Orlov, and L. Acho, "An invariance principle for discontinuous dynamic systms with application to a Coulomb friction oscillator," J. Dyna. Syst., Measure., Contol, vol. 122, pp. 687-690, 2000.

[20] B. P. Demidovich, The Dissipativity of a Nonlinear System of Differential Equations (in Russian). Moscow, U.S.S.R.: Vestnik Moscow State Univ., 1961, 1962, vol. 6, 1, Matem. Mekh, pt. I, II, pp. 19-27.

[21] _ Lectures on Mathematical Theory of Stability (in Russian). Moscow, U.S.S.R.: Nauka, 1967.

[22] A. Pavlov, A. Pogromsky, N. van de Wouw, and H. Nijmeijer, "Convergent dynamics, a tribute to Boris Pavlovich Demidovich," Syst. Control Lett., vol. 52, pp. 257-261, 2004.

[23] T. Yoshizawa, Stability Theory by Liapunov's Second Method. Tokyo, Japan: Math. Soc. Japan, 1966.

[24] W. Lohmiller and J.-J. E. Slotine, "On contraction analysis for nonlinear systems," Automatica, vol. 34, pp. 683-696, 1998.

[25] D. Angeli, "A Lyapunov approach to incremental stability properties," IEEE Trans. Autom. Control, vol. 47, no. 3, pp. 410-421, Mar. 2002.

[26] V. Fromion and G. Scorletti, "Analysis of nonlinear systems by their linearizations," in Proc. 41 st IEEE Conf. Decision Control, Las Vegas, NV, Dec. 2002, pp. 2669-2674.

[27] A. Y. Pogromsky, G. Santoboni, and H. Nijmeijer, "An ultimate bound on the trajectories of Lorenz system and its applications," Nonlinearity, vol. 16, pp. 1597-1605, 2003.

[28] A. Eden, "Local Lyapunov exponents and a local estimate of Hausdorff dimension," Math. Model. and Numer. Anal., vol. 23, no. 3, pp. 405-413, 1989.

[29] A. S. Ljashko, "Estimates for Fractal and Hausdorff Dimensions of Invariant Sets of Dynamical Systems," Ph. D., Dept. Math. Mech., St. Petersburg State Univ., St. Petersburg, Russia, 1994.

[30] G. A. Leonov, "Lyapunov dimension formulas for Fenon and Lorenz attractors," St. Petersburg Math. J., vol. 13, pp. 1-12, 2001.

[31] G. A. Leonov, I. M. Burkin, and A. I. Shepeljavyi, Frequency Methods in Oscillation Theory. Dordrecht, The Netherlands: Kluwer, 1996.

[32] G. A. Leonov, "On a method for investigating the global stability of nonlinear systems," in Vestnik Leningrad Univer.. St. Petersburg, Russia: St. Petersburg Univ., 1991, vol. 4, Mat. Mekh. Astron., pp. $11-14$.

[33] H. Federer, Geometric Measure Theory. New York: Springer-Verlag, 1969

[34] F. Morgan, Geometric Measure Theory, 2nd ed. San Diego: Academic, 1995

[35] R. Courant, Dirichlet's Principle, Conformal Mappings and Minimal Surfaces. New York: Wiley-Interscience, 1950.

[36] F. R. Gantmacher, Applications of the Theory of Matrices. New York: Wiley-Interscience, 1959.

[37] R. Leine and H. Nijmeijer, Dynamics and Bifurcations of Non-Smooth Mechanical Systems. New York: Springer-Verlag, 2004, vol. 18, Lecture Notes in Applied and Computational Mechanics.

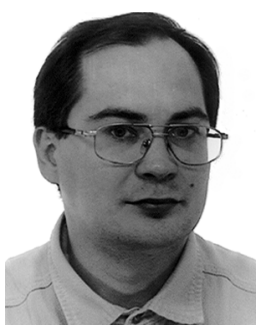

Alexander Pogromsky received the M.Sc. degree from the Baltic State Technical University, Russia, in 1991, and the Ph.D. (Candidate of Science) degree from the St. Petersburg Electrotechnical University, St. Petersburg, Russia, in 1994.

From 1995 till 1997, he was with the Laboratory "Control of Complex Systems" (the Institute for Problems of Mechanical Engineering, St. Petersburg, Russia). From 1997 to 2000, he was a Research Fellow with the Department of Electrical Engineering, Division on Automatic Control, Linkoping 
University, Sweden, and the Department of Electrical Engineering, Eindhoven University of Technology, The Netherlands. Currently, he is an Assistant Professor in the Mechanical Engineering Department of Eindhoven University of Technology. His research interests include theory of nonlinear, adaptive and robust control, and nonlinear oscillations.

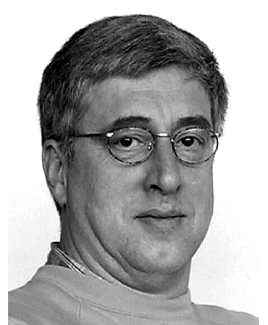

Henk Nijmeijer (F'99) received the M.Sc. and $\mathrm{Ph} . \mathrm{D}$. degrees in mathematics from the University of Groningen, Groningen, The Netherlands, in 1979 and 1983 , respectively.

From 1983 till 2000, he was affiliated with the Department of Applied Mathematics of the University of Twente, Enschede, The Netherlands. Since 1997, he was also part-time affiliated with the Department of Mechanical Engineering of Eindhoven University of Technology, Eindhoven, The Netherlands. Since 2000, he has been a Full Professor at Eindhoven, and chairs the Dynamics and Control Group. He published a large number of journal and conference papers, and several books, including the "classical" Nonlinear Dynamical Control Systems (New York: Springer-Verlag, 1990), Synchronization of Mechanical Systems (Singapore, World Scientific, 2003), Dynamics and Bifurcations of Non-Smooth Mechanical Systems (New York: Springer-Verlag, 2004), and Uniform Output Regulation of Nonlinear Systems (Norwell, MA: Birkhauser, 2005).

Dr. Nijmeijer is Editor-in-Chief of the Journal of Applied Mathematics, Corresponding Editor of the SIAM Journal on Control and Optimization, and board member of the International Journal of Control, Automatica, European Journal of Control, Journal of Dynamical Control Systems, SACTA, International Journal of Robust and Nonlinear Control, International Journal on Bifurcation and Chaos, and the Journal of Applied Mathematics and Computer Science. He was awarded the IEE Heaviside Premium in 1990. He has been involved in the organisation of several International Conferences, like the IFAC Nonlinear Control Symposium (NOLCOS), Enschede 1998 (IPC-chair and organiser), Workshop on "New Directions in Nonlinear Observer Design," Geiranger, 1999 (co-chair), Workshop on Group Coordination and Cooperative Control, Tromso, 2006 (co-chair), IFAC Symposium Chaos, Reims, 2006 (IPC-co-chair).

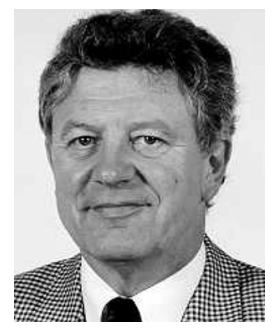

Jacobus Rooda (M'90) received the M.S. degree from Wageningen University of Agriculture Engineering and the $\mathrm{Ph} . \mathrm{D}$. degree from Twente University of Technology, The Netherlands, in 1971 and 1978, respectively.

Since 1985, he has been a Professor of (Manufacturing) Systems Engineering at the Department of Mechanical Engineering, Eindhoven University of Technology, The Netherlands. His research fields of interest are modeling and analysis of manufacturing systems. His interest is especially in control of manufacturing lines and in supervisory control of manufacturing machines. 